$\xi=-1$

\title{
Health monitoring of floating structure by utilizing support vector machines
}

\author{
Akihisa Tabata * \\ Department of Precision Machinery Engineering, Nihon University \\ *Corresponding author E-mail: tabata@eme.cst.nihon-u.ac.jp
}

\begin{abstract}
We explored the damage detection method for a floating structure by utilizing the natural ocean waves for the floating body. And we utilized the discrete Fourier transform as well as the learning and judgment functions of the support vector machines. It showed a possibility that it can perform the health diagnosis for the floating structure even using the strain response vibrations by inputting the natural waves with different cycles and wave heights.
\end{abstract}

Keywords: Remote Monitoring; Support Vector Machines; Piezoelectric Films.

\section{Introduction}

At present, there are a lot of problems to solve, such as the submerged land problem arising from the sea level rise due to climate change, land issue due to population growth, and energy problem having become prominent after the Great East Japan Earthquake. There are various efforts to solve such problems, and as one of them, the usage of marine space by using a large floating structure is expected [1], [2], [3]. However, in order to realize a very large floating structure, there are a number of issues such as cost required in the operation/management [4] and ensuring the safety at the time of maintenance [5], [6], [7]. To solve these problems, a low-cost, safe and automated operation/management method without relying on humans is sought. Therefore, in this study, we explored the damage detection method for a floating structure by utilizing the natural ocean waves for the floating body installed offshore and receiving the waves at all times with an aim to build a full-time maintenance system capable of operating it in safe and at low-cost. Moreover, in the ocean waves utilized for the excitation of the floating structure, there is some variation in the cycle or wave height. So in order to perform accurately the damage detection, we utilized the discrete Fourier transform as well as the learning and judgement functions of the support vector machines.

\section{Measurement of the strain response vibra- tion of a floating body}

In the damage detection experiment of this study, a flat water tank for ocean experiment $(27 \mathrm{~m} \times 7 \mathrm{~m})$ installed in the Techno Place of Funabashi school building of the College of Science and Technology, Nihon University was used. By creating different states when no damage is present (normal) and when a damage is progressing (abnormal) with a floating body model floated on the water surface of the water tank, waves generated by waveproducing equipment were input for the respective states. The vibrational responses of the mega-float for the waves with a varia- tion in the cycle and wave height were measured by the piezo film sensor to perform a frequency analysis.

\subsection{Experimental floating body model}

Since the large floating structure can be regarded as a very flat structure, for the rectangular plate made of material SPHC, a polystyrene foam is attached to the bottom surface as a float to moor it via a linear spring at 4 corners, having constructed a $1 / 1000$ scale model of the actual equipment. Table 1 shows the main dimensions and the like.

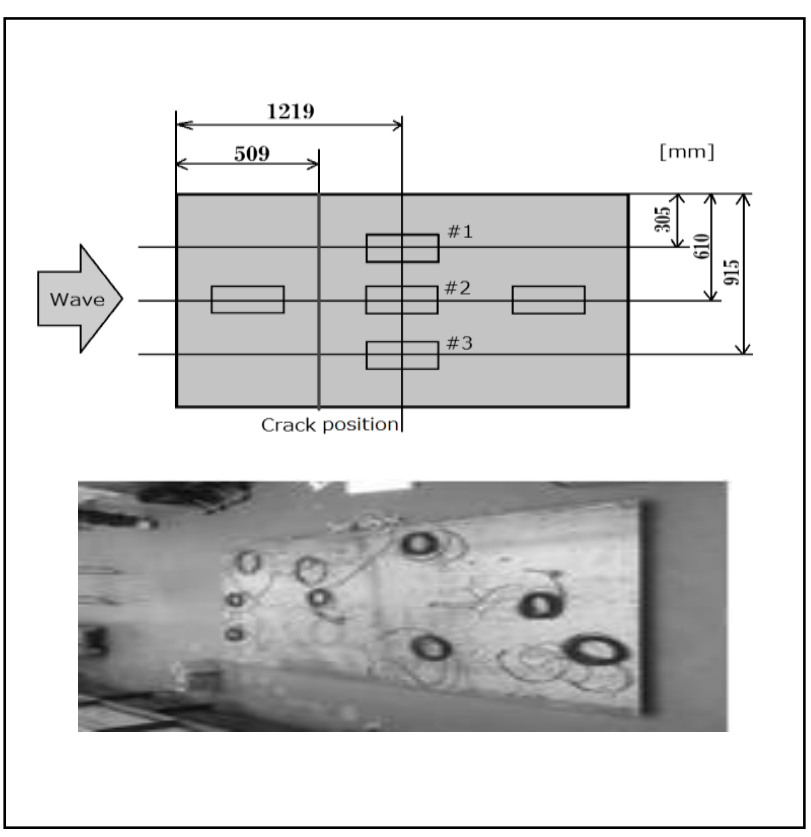

Fig. 1: A Floating Body Model and Sensor Setting Positions. 
Table 1: Dimensions of the Floating Body

\begin{tabular}{lllll}
\hline Material & Length [mm] & Width [mm] & Thickness [mm] & Mass [kg] \\
\hline SPHC & 2420 & 1200 & 12 & 20 \\
\hline
\end{tabular}

\subsection{Piezo film sensor}

The piezo film sensor is a polymer film made from PVDF (polyvinylidene fluoride) having a piezoelectric effect, good in workability, a piezoelectric element easy to make a large area and thinning, and a sensor to measure the load or strain applied to the piezoelectric film by changing it into an electric signal. Because it is a power generation element, there is no need to supply power to the sensor itself. Moreover, it has excellent flexibility and impact resistance, high voltage resistance, water resistance and chemical stability. The greatest features of the piezoelectric film are frequency property capable of capturing a wide range of frequency bands and difficult to have a specific natural frequency because it is light and soft, having both features of impact resistance and robustness. While it is possible to capture small response, there is a problem to pick up noise, making it difficult to analyze the phenomenon.

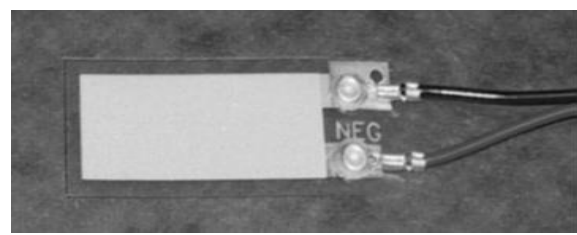

Fig. 2: Piezo Film Sensor.

\subsection{Experimental method}

Experiments were conducted by setting the water depth $\mathrm{h}$ to 80 $\mathrm{mm}$ and the height of the wave to $10 \mathrm{~mm}$, setting the regular wave cycles to $0.9 \mathrm{~s}, 1.1 \mathrm{~s}, 1.3 \mathrm{~s}$ to make 3 patterns, and also giving a cut from both sides by $0 \mathrm{~mm}, 200 \mathrm{~mm}, 400 \mathrm{~mm}$, respectively to make 9 patterns in total. As an example, we entered $1.1 \mathrm{~s}$ for the regular wave cycle.

\subsection{Measurement conditions and the frequency analysis method}

We assumed that there occurs a difference in the strain response vibration between the normal time when no damage is present and the abnormal time when a damage is given, utilized piezoelectric film for the measuring apparatus, and set the sampling frequency to $100 \mathrm{~Hz}$.And then, we performed the signal processing for the measured time series signals of the strain response vibration by using a discrete Fourier transform.

\section{Application of the support vector machines}

The support vector machines is originally a linear discriminator, and the discriminant function can be expressed by Eq. (1) by letting the input vector be xi and the output be $\mathrm{y}_{\mathrm{i}}$

$y_{i}=w^{T} x_{i}+b$

Where $\mathrm{w}$ is a weight vector and $\mathrm{b}$ is a bias term, and $\mathrm{i}$ represents the data number and $\mathrm{T}$ represents the transpose of matrix.

The support vector machines has a feature in the learning method: when there is a population of sample data $\mathrm{x}_{\mathrm{i}}$ belonging to 2 classes, the nearest data in each other class are selected, and a margin like a band as shown by a broken line in Fig. 3 is set so that the distance between the data becomes maximum to set the hyperplane to become the boundary of the 2 classes at its center.

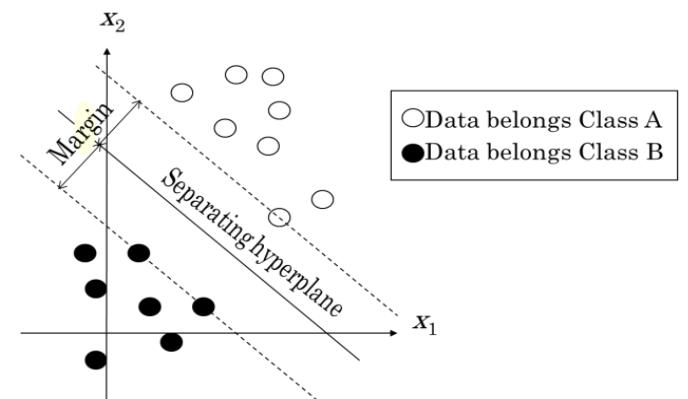

Fig. 3: Discriminant Hyperplane of the Support Vector Machines.

Where, the minimum distance between the learning data xi and the discriminant hyperplane can be expressed by Eq. (2).

$\min _{i=1 \ldots 1} \frac{\left|w^{T} x_{i}+b\right|}{\|w\|}$

Because this distance should be maximized, the learning of the support vector machines for seeking the discriminant hyperplane can be realized by solving the optimization problem of the following equation to obtain a weight vector $\mathrm{w}$.

$\begin{array}{ll}\text { minimize } & \|\mathrm{w}\|^{2} \\ \text { subject to } & \mathrm{y}_{\mathrm{i}}\left(\mathrm{w}^{\mathrm{T}} \mathrm{x}_{\mathrm{i}}+\mathrm{b}\right) \geq 1\end{array}$

This method is said to be a method capable of most accurately estimating the discriminant boundary of the population belonging to 2 classes from the sample data based on the statistical learning theory [8].

Because the support vector machines is originally a linear discriminator, only one-lower dimensional discriminant plane than the dimension number of the data can be obtained. Therefore, the distance between each other learning data is also used for learning to realize a non-linear discrimination. This extension to the nonlinear discrimination $[9,10]$ is to replace the data vector using a kernel function as expressed by Eq. (4) and to perform the learning and judgement using the replaced data vector.

$K\left(x_{j}, x_{i}\right)=x_{j}^{T} x_{i}$

Where $x_{j}$ is the vector containing all the sample data, and the nonlinear function expressed by Eq. (4) is called as polynomial kernel. By using such a non-linear discriminant function having been learned based on the sample data, we detected the degree of damage from the frequency analysis result of the measured strain response vibration.

\section{Features of the measurement signal}

Figures 4,5 and 6 show the measurement signals by the sensors $\# 1$, \#2 and \#3 (Fig. 1) attached to the left, center and right sides for the incident wave direction from the floating body side for the regularly input wave of cycle 1.1 , by converting them using the discrete Fourier transform. Changes in the response of low frequency components of $2 \mathrm{~Hz}$ or less were remarkable. 


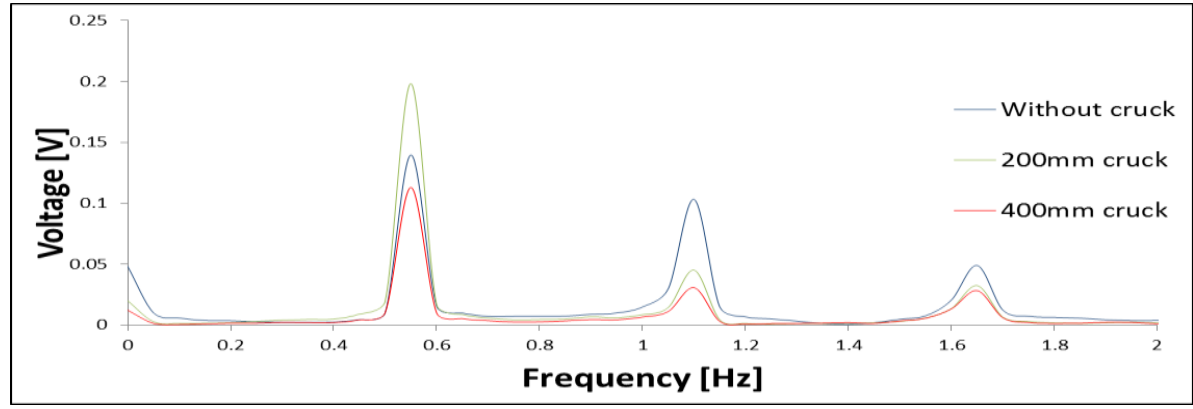

Fig. 4: Discrete Fourier Transform Result of the Measurement Signal of Sensor \#1.

\begin{tabular}{llll}
\multicolumn{4}{c}{ Table 2: Second Peak Value of Sensor \#1 Signal } \\
\hline Frequency & Without Crack & $200 \mathrm{Mm}$ Crack & $400 \mathrm{Mm}$ Crack \\
\hline $1.1 \mathrm{~Hz}$ & $0.103 \mathrm{~V}$ & $0.0452 \mathrm{~V}$ & $0.0308 \mathrm{~V}$ \\
\hline
\end{tabular}

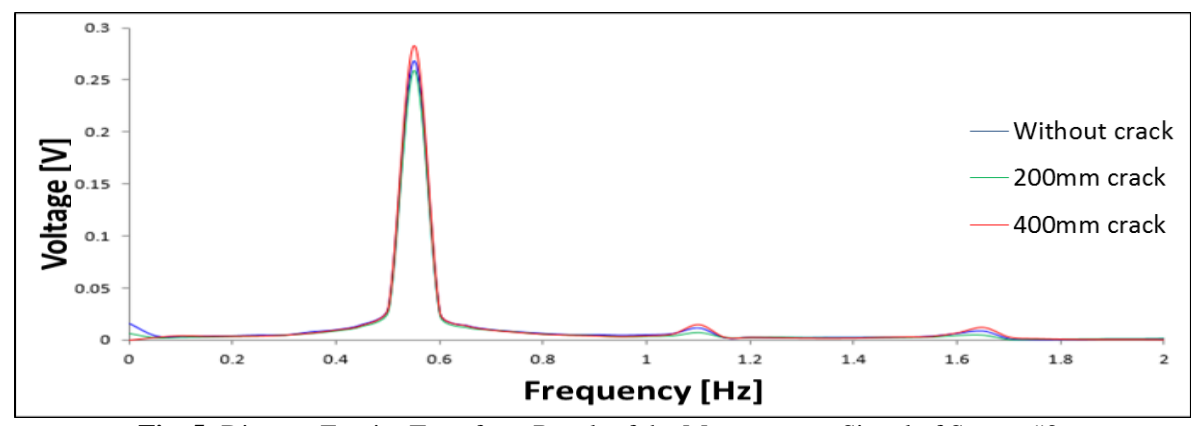

Fig. 5: Discrete Fourier Transform Result of the Measurement Signal of Sensor \#2.

Table 3: Second Peak Value of Sensor \#2 Signal

\begin{tabular}{llll} 
& & \\
\hline Frequency & Without Crack & $200 \mathrm{~mm}$ Crack & $400 \mathrm{~mm} \mathrm{Crack}$ \\
\hline $0.55 \mathrm{~Hz}$ & $0.268 \mathrm{~V}$ & $0.259 \mathrm{~V}$ & $0.283 \mathrm{~V}$ \\
\hline
\end{tabular}

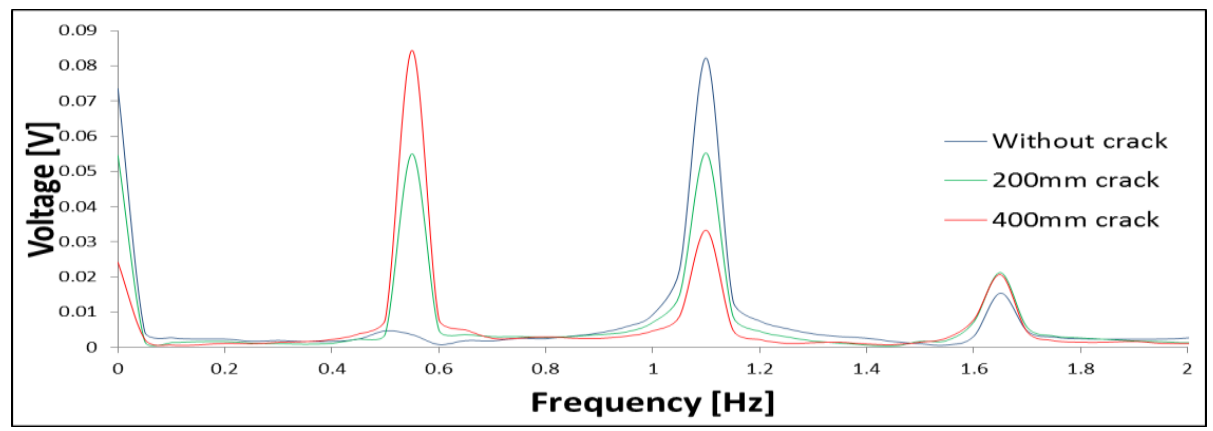

Fig. 6: Discrete Fourier Transform Result of the Measurement Signal of Sensor \#3.

Table 4: Second Peak Value of Sensor \#3 Signal

\begin{tabular}{llll}
\hline Frequency & Without Crack & $200 \mathrm{~mm}$ Crack & $400 \mathrm{~mm}$ Crack \\
\hline $0.55 \mathrm{~Hz}$ & $0.604 \mathrm{~V}$ & $0.335 \mathrm{~V}$ & $0.0248 \mathrm{~V}$
\end{tabular}

From Fig. 4, a tendency was observed that the signal intensity of sensor \#1 decreased around $1.1 \mathrm{~Hz}$ depending on the depth of the crack. This is considered to be caused by a fact that the floating body becomes a state such as that each part of it moves independently in the vicinity of the crack portion by enlarging the damage, resulting in decreasing the strain at the targeted sensor installation position.

Because variation occurs in the signal intensity by the frequency in the damages of $0 \mathrm{~mm}$ and $200 \mathrm{~mm}$ from Fig. 5, it is considered to be difficult to find the difference. However, a tendency to increase in $400 \mathrm{~mm}$ was observed in all the results. In the closest part from the crack portion, in the case of small crack from $0 \mathrm{~mm}$ to $200 \mathrm{~mm}$, the effect due to the cyclical change of the waves is larger and the signal strength changes depending on the cycle, making it hard to distinguish the difference unless the size of the crack expands as large as $400 \mathrm{~mm}$.

From Fig. 6, also for sensor \#3, similar to case of sensor \#1, it is to be caused by a fact that the floating body becomes a state such as that each part of it moves independently in the vicinity of the crack portion at which the signal intensity is observed to decrease around $1.1 \mathrm{~Hz}$, resulting in decreasing the strain at the targeted sensor installation position.

In the symmetrically located right and left sensors, the same tendency was observed at the time of the second peak.

\section{Damage detection by the support vector machines}

For the strain response vibration measured using a piezo film sensor, crack detection experiment by the nonlinear support vector machines was carried out using the frequency-signal strength peak value by the result of the discrete Fourier transform. We used the sensor signals that were simultaneously measured using the piezo film sensors attached at 3 positions to perform the learning and estimation of the position causing the crack and its size. The results are shown in Table 5. 
Table 5: Clack Estimation Result

\begin{tabular}{llll}
\hline Estimation item & Without crack & $200 \mathrm{~mm}$ crack & $400 \mathrm{~mm}$ crack \\
\hline Crack position & - & $483 \mathrm{~mm}$ & $512 \mathrm{~mm}$ \\
Crack size & 0 & $193 \mathrm{~mm}$ & $387 \mathrm{~mm}$ \\
\hline
\end{tabular}

The crack positions in Table 5 show the distance from the left end of the floating body model of Fig. 1. In addition, this crack estimation result was the estimated mean value by the 9-times experimental data obtained by the input of waves by changing the cycle and height in the large water tank.

Both the position of crack and its size can be estimated with sufficient accuracy. Moreover, the data used in this experiment is obtained by applying the discrete Fourier transform to the strain response vibration measured at 30 seconds after inputting the waves for the floating body model in the water tank. Therefore, the strain response vibration is learned initially when the floating body is moored on the sea, thereafter the measurement is continued, and by inputting the signal to the discriminator of the support vector machines to make it judge the crack, it is believed that the support vector machines can help to conserve the floating body by early detecting the damage occurred on it.

\section{Conclusion}

Frequency analysis by the discrete Fourier transform was carried out for the measurement signals of the piezo film sensors attached to the floating body to diagnose the location and size of the damage by applying the non-linear support vector machines to that data.

As a result, it showed a possibility that it can perform the health diagnosis for the floating structure even using the strain response vibrations by inputting the natural waves with different cycles and wave heights. This is useful for the introduction of structural health diagnosis utilizing the renewable energy.

\section{Acknowledgement}

The author would like to thank the Department of Precision Machinery Engineering Nihon University.

This research was funded by the Nihon University.

\section{References}

[1] Tomoki Ikoma, Koichi Masuda, Yuka Watanabe and Hisaaki Maeda, "Hydroelastic Responses and Performance of OWC Type WECs of a Large Scale Floating Structure", Proceedings of the sixth International Conference on Hydroelasticity in Marine Technology, Tokyo, 2012, pp:445-455.

[2] H. Osawa, Y. Washio, T. Miyazaki, T. Hotta and T. Miyazaki, "R\&D of Technologies of Wave Energy Application- Development of Offshore Floating Wave Power Device named Mighty-Whale-", JAMSTEC, 2004.

[3] J.A. Pinkster and E.J.A. Meevers Scholte, "The behaviour of a large air-supported MOB at sea", Proceedings of the 3rd International Workshop on Very Large Floating Structures, VLFS '99, Vol. II, pp:567-576, 1999.

[4] Tomoki Ikoma, Koichi Masuda, Hikaru Omori, Hiroyuki Osawa and Hisaaki Maeda, "Improvement of Wave Power Take-Off Performance due to the Projecting Walls for OWC Type WEC", Pro ceedings of the ASME 32nd International Conference on Ocean, Offshore and Arctic Engineering (OMAE'13), ASME, 10384, 2013.

[5] H. Maeda, C.H. Rheem, Y. Washio, H. Osawa, Y. Nagata, T. Ikoma, N. Fujita and M. Arita, "Reduction Effects of Hydroelastic Responses on a Very Large Floating Structure With Wave Energy Absorption Devices Using OWC System", Proceedings of the 20th International Conference on OMAE 2001, ASME, CD-ROM file is OSU-5013, 2001.6.

[6] Hiroaki Eto, Chiaki Sato, Koichi Masuda, Tomoki Ikoma, Ken Shimizu, Akio Kuroyanagi, Akio Kobayashi, Sachio Togawa, Sotaro Tsuboi, Kosaku Kinoshita, Junko Yamaguchi, Toshikatsu Saito, Masako Takada, and Atsuko Tanigome, "Feasibility Study of MEDI-FLOAT installed in the River", Proceedings of Pacific Con- gress on Marine Science \& Technology (PACON) 24th International Conference, 2014.

[7] Kentaro Tsuji, Kazuhisa Naoi, Mitsuhiro Shiono, Katsuyuki Suzuki, "Study on the Gear Ratio for a Tidal Current Power Generation System Using the MPPT Control Method", Proceedings of the Twenty-fourth (2014) International Ocean and Polar Engineering Conference, 2014, pp:568-574.

[8] Vladimir N. Vapnik, Statistical Learning Theory, WileyInterscience, (1998).

[9] B. Schölkopf, P. Simard, A. Smola, and V. Vapnik, "Prior knowledge in support vector kernels", Advances in Neural Information Processing Systems 10, pp.640-646, 1997.

[10] D. Decoste, "Training invariant support vector machines", Machine Learning, vol. 46, pp. 161-190, 2002.education on Internet. Patient Education and Counseling 53, 309-313. 